\title{
ANTI-SYMMETRY AND NON-EXTENSIONAL MEREOLOGY
}

\author{
By Aaron J. Cotnoir
}

I examine the link between extensionality principles of classical mereology and the anti-symmetry of parthood. Varzi's most recent defence of extensionality depends crucially on assuming anti-symmetry. I examine the notions of proper parthood, weak supplementation and non-well-foundedness. By rejecting anti-symmetry, the anti-extensionalist has a unified, independently grounded response to Varzi's arguments. I give a formal construction of a non-extensional mereology in which antisymmetry fails. If the notion of 'mereological equivalence' is made explicit, this non-anti-symmetric mereology recaptures all of the structure of classical mereology.

In the most recent and extended defence of extensionality principles in mereology, Achille Varzi argues that challenges to extensionality principles are either selfdefeating or unsupported. ${ }^{1}$ Varzi claims that his main argument does not presuppose the anti-symmetry of parthood. In this paper, I show that it does presuppose the anti-symmetry of parthood. Towards this end, I develop a non-extensional mereology in which anti-symmetry fails. This new mereology has the additional benefit of recapturing classical mereological structure, given natural constraints.

\section{EXTENSIONALITY OF PARTHOOD}

Mereology is the formal theory of the relationship between parts and wholes. According to classical mereology, the extensionality of parthood principle (EP) is a criterion for the identity of objects:

EP. If $x$ and $y$ are composite objects with the same proper parts, then $x=y$.

The principle is so named after the parallel extensionality principle of set theory: two sets are identical if and only if they have all the same members. In mereology, the principle is restricted only to composite objects, objects that have proper parts, so as to allow for more than one uncomposed object (called 'atoms'). In most mereologies, $(\mathrm{EP})$ is derived as a theorem following from the strong supplementation principle: if $x$ is not part of $y$, then there is some $z$ that is part of $x$ but disjoint from $y$. Given the

${ }^{1}$ A.C. Varzi, 'The Extensionality of Parthood and Composition', The Philosophical Quarterly, $5^{8}$ (2008), pp. 108-33.

(C) 2009 The Author Journal compilation (C) 2009 The Editors of The Philosophical Quarterly

Published by Blackwell Publishing, 9600 Garsington Road, Oxford ox4 2DQ, UK, and 350 Main Street, Malden, MA 02I48, USA 
identity of objects $x$ and $y$ in accordance with (EP), the indiscernibility of identicals entails that everything true of $x$ must also be true of $y$ and vice versa. This result has led many to reject extensionality principles, including (EP). For example, it is natural to think that a lump of clay and a statue made from it have all the same proper parts. But is everything true of the clay also true of the statue? One standard objection suggests that while the lump of clay can survive being completely flattened, the statue cannot. If the indiscernibility of identicals has to stay, then (EP) must go.

\section{I.I. Varzi on $(E P)$}

Against such standard objections, Achille Varzi advances a new argument for (EP). The argument is a reductio of the anti-extensionalist position; it begins by considering two distinct composite objects $\alpha$ and $\beta$ such that

I. $\alpha \neq \beta$.

In order for these two objects to be a counter-example to (EP), they must be mereologically indiscernible in the sense given by

2. $\alpha$ and $\beta$ have all the same proper parts.

Given classical logic, which governs our mereology, one of the following is true. ${ }^{2}$

3. $\beta$ is part of $\alpha$

4. $\beta$ is not part of $\alpha$.

According to Varzi, the anti-extensionalist is now in a dilemma. Whichever of (3) or (4) the anti-extensionalist chooses, we can go on to show that $\alpha$ and $\beta$ do not have all the same proper parts, contradicting (2). The conclusion which follows from acceptance of (4) is nuanced, and the arguments for it are complicated. For present purposes, I focus attention on (3), since this is the horn of the dilemma that I reject. Varzi's argument (p. I 6 , numbering adjusted) from (3) to the negation of (2) is as follows:

Suppose we accept (3), as Aristotle and many others since have suggested. Then $\beta$ would have to be a proper part of $\alpha$, because of (I). But surely $\beta$ is not a proper part of itself - nothing is. So $\alpha$ and $\beta$ would not be mereologically indiscernible after all: the former would, while the latter would not, include $\beta$ among its proper parts. Hence (2) would be false. If (2) is false, $(\mathrm{EP})$ is vacuously safe.

The inference has three steps: (a) the inference from (3) to $\beta$ 's being a proper part of $\alpha$; (b) the fact that the proper parthood relation is well founded; ${ }^{3}$ and (c) the inference to mereological discernibility. The apparent straightforwardness of the argument leads Varzi to claim (ibid.) that the argument in no way depends on the antisymmetry of parthood:

This argument does not presuppose the anti-symmetry of parthood. As I have mentioned, I take it that anti-symmetry is one of the minimal requirements which any

${ }^{2}$ However, see N.J.J. Smith, 'A Plea For Things That Are Not Quite All There: or, Is There a Problem about Vague Composition and Vague Existence?', Fournal of Philosophy, IO2 (2005), pp. 38I-42I, for a mereology based in a logic for which excluded middle fails.

3 A strict order < (in this case proper parthood) on a domain is said to be well founded if every subset of that domain has a $<$-minimal element. 
relation must satisfy in order to qualify as parthood, but some may feel otherwise. In particular, the anti-extensionalist who is willing to accept (3) might also be inclined to accept its converse

5. $\alpha$ is part of $\beta$

while insisting on (3). Pretty clearly, this is not enough to block the argument, which could now be run twice.

But this is false. The argument above does depend on the anti-symmetry of parthood, particularly in step (a). In what follows, I argue that an anti-extensionalist who denies anti-symmetry will have independent resources for avoiding Varzi's argument.

\section{I.2. Proper parthood}

To show how the inference in step (a) depends on the anti-symmetry of parthood, I must first look more closely at proper parthood. There are two standard candidates. ${ }^{4}$

PPI. $x$ is a proper part of $y \equiv_{d f} x$ is part of $y$ and $x \neq y$

$\mathrm{PP}_{2}$. $x$ is a proper part of $y \equiv_{d f} x$ is part of $y$ and $y$ is not part of $x$.

In classical mereology, $\left(\mathrm{PP}_{1}\right)$ and $\left(\mathrm{PP}_{2}\right)$ are equivalent. In mereologies without the anti-symmetry axiom, however, the equivalence may break down. The details of Varzi's argument suggest that he had (PPI) in mind. ${ }^{5}$ Given (I) and (3), we have it that $\alpha \neq \beta$ and $\beta$ is part of $\alpha$; thus we have both conjuncts of (PPI). It follows immediately that $\beta$ is a proper part of $\alpha$.

This is true so long as proper parthood is defined by (PPI). The antiextensionalist who accepts that $\alpha$ and $\beta$ are parts of each other - that is, accepts (3) and its converse - will not avoid contradicting (2). This is because the argument can now be run a second time: from (I) and the converse of (3), we have it that $\alpha$ is a proper part of $\beta$ by $(\mathrm{PPI})$. Also from $(\mathrm{PPI})$ we have it that $\beta$ is not a proper part of $\beta$, since $\beta=\beta$. Thus even without anti-symmetry, the anti-extensionalist contradicts (2).

However, the situation changes if proper parthood is defined by $\left(\mathrm{PP}_{2}\right)$. In that case, the inference in step (a) will not go through unless we presuppose the antisymmetry of parthood. Even if we have (3), that $\beta$ is part of $\alpha$, we also need that $\alpha$ is not part of $\beta$ in order to infer by $\left(\mathrm{PP}_{2}\right)$ that $\beta$ is a proper part of $\alpha$. How do we get this? Anti-symmetry ensures that if (I) $\alpha \neq \beta$ and (3) $\beta$ is part of $\alpha$, then $\alpha$ is not part of $\beta$. But if the anti-extensionalist rejects anti-symmetry and admits that $\alpha$ is part of $\beta$, we cannot derive that $\beta$ is a proper part of $\alpha$ according to $\left(\mathrm{PP}_{2}\right)$. So the antiextensionalist is not forced to contradict (2), and therefore does have a viable counter-example to extensionality.

${ }^{4}$ For the reader who doubts the standardness of $\left(\mathrm{PP}_{2}\right)$, a glance at the literature shows that both definitions have seen recent use. For example, Varzi himself uses $\left(\mathrm{PP}_{2}\right)$ in his Stanford Encyclopedia entry: Varzi, 'Mereology', in E.N. Zalta (ed.), The Stanford Encyclopedia of Philosophy, http://plato.stanford.edu/entries/mereology (see def. 2I). R. Casati and A.C. Varzi, Parts and Places: the Structures of Spatial Representation (MIT Press, I999), likewise use ( $\left.\mathrm{PP}_{2}\right)$ (see def. 3.3, p. 36). As for (PPI), P.M. Simons, Parts: a Study in Ontology (Oxford: Clarendon Press, I987), p. II, uses it; so do H.S. Leonard and N. Goodman, 'The Calculus of Individuals and its Uses', Fournal of Symbolic Logic, 5 (i940), pp. 45-55 (see def. I.or I, p. 47). In other mereologies, proper parthood is taken as primitive and general parthood is defined from it.

${ }^{5}$ In conversation, Varzi has informed me that (PPI) is indeed the definition presupposed. 
It is open to the defender of extensionality principles to give a philosophical argument, as opposed to a formal mereological argument, to the effect that antiextensionalists contradict (2). One might argue that a unified whole like $\alpha$ cannot be part of the matter of which it is composed, namely, $\beta$. If this argument succeeds, then the converse of (3) does not hold, and thus the anti-extensionalist is still stuck in Varzi's dilemma. ${ }^{6}$ The argument, however, would need to be made explicit. Here I simply note that any such argument would amount to a defence of the anti-symmetry of parthood. Naturally, then, an anti-extensionalist who denies anti-symmetry will have to reply.

I suspect that at this point the reader is wondering why anyone should prefer $\left(\mathrm{PP}_{2}\right)$ rather than $(\mathrm{PPI})$. I contend that there are two important reasons why an antiextensionalist who denies anti-symmetry will prefer $\left(\mathrm{PP}_{2}\right)$ to $(\mathrm{PP} \mathrm{I})$. First, $(\mathrm{PPI})$, but not $\left(\mathrm{PP}_{2}\right)$, is inconsistent with weak supplementation. Secondly, $(\mathrm{PP})$, but not $\left(\mathrm{PP}_{2}\right)$, is a non-well-founded relation. I discuss each reason in turn.

The first reason to prefer $\left(\mathrm{PP}_{2}\right)$ over $(\mathrm{PPI})$ is that the latter is inconsistent with the principle of weak supplementation (WS) if anti-symmetry is rejected.

WS. If $x$ is a proper part of $y$, then there is some $z$ that is part of $y$ and disjoint from $x$.

(WS) has been considered by many to be constitutive of the concept of proper parthood. ${ }^{7}$ Strong supplementation is usually blamed for extensionality principles, and (WS) is often considered uncontroversial even for anti-extensionalists. ${ }^{8}$ The problem, however, is that (PPI) is inconsistent with (WS). In the candidate counter-example to (EP) above, $\alpha$ and $\beta$ are mutual parts but not identical. Then according to (PPI), $\alpha$ and $\beta$ must be mutual proper parts. By (WS), this means there must be a part of $\alpha$ disjoint from $\beta$ and a part of $\beta$ disjoint from $\alpha$. But given the transitivity of parthood, this is contrary to the supposition that $\alpha$ and $\beta$ are mutual parts. So by endorsing (PPI), an anti-extensionalist who denies anti-symmetry would be forced to do without an important and extensionality-neutral axiom of mereology, namely, (WS).

$\left(\mathrm{PP}_{2}\right)$, in contrast, is perfectly consistent with (WS). In the model represented as a directed graph in Figure I, indexed letters are objects and directed lines are to be read as the parthood relation. ${ }^{9}$ This is a model of a non-anti-symmetric parthood relation that, assuming $\left(\mathrm{PP}_{2}\right)$, satisfies (WS). On $\left(\mathrm{PP}_{2}\right), b_{1}$ and $b_{2}$ are both proper parts of $a_{1}$, and each is a proper part of $a_{2}$. It is to

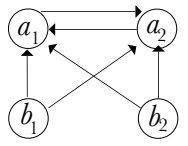

Figure I be noted that $b_{1}$ and $b_{2}$ are disjoint: they have no parts in common. For each proper part of $a_{1}$, there is another disjoint proper part; in the case of $b_{1}, b_{2}$ is the second

${ }^{6}$ Thanks to Achille Varzi for suggesting this line of response.

${ }^{7}$ For example, see Simons, 'Free Part-Whole Theory', in his Philosophical Applications of Free Logic (Oxford UP, I99I), pp. 285-306. For examples of those who reject (WS), see P. Forrest, 'Non-Classical Mereology and Its Application to Sets', Notre Dame Fournal of Formal Logic, 43 (2002), pp. 79-94; D. Smith, 'Mereology without Weak Supplementation', Australasian Journal of Philosophy (forthcoming).

${ }^{8}$ Importantly, however, see Varzi, 'Universalism Entails Extensionalism', Analysis (forthcoming).

${ }^{9}$ Such figures are often given as Hasse diagrams. But Hasse diagrams represent partial orders. I thus revert to a directed graph to represent the parthood preorder. 
proper part of $a_{1}$, and in the case of $b_{1}, b_{2}$ is the second proper part. Also, for each proper part of $a_{2}$ we have the same result. In consequence, even if Varzi's argument succeeds when (PPI) is presupposed, it merely defeats an unnecessarily weak version of the position.

The above example leads to the second reason to prefer $\left(\mathrm{PP}_{2}\right)$ to $(\mathrm{PP})$. According to (PPI), $a_{1}$ must be a proper part of $a_{2}$, and $a_{2}$ must be a proper part of $a_{1}$. Therefore the proper parthood relation is not asymmetric, and therefore it is not a well founded relation. But Varzi's argument uses the fact that proper parthood is a well founded relation in step (b). It is not clear why an anti-extensionalist who endorses a non-well-founded proper parthood relation is forced to accept that nothing is a proper part of itself. ${ }^{10}\left(\mathrm{PP}_{2}\right)$ is a well founded relation, because it is by definition asymmetric and irreflexive. ${ }^{11}$

The upshot, then, is that if Varzi's argument uses $\left(\mathrm{PP}_{2}\right)$, then either it presupposes anti-symmetry, or it fails. But if the argument uses (PPI), even if it is successful, it merely defeats a straw man.

\section{I.3. Anti-symmetry}

Is rejection of the anti-symmetry axiom just an ad hoc escape from Varzi's argument? What reasons might the anti-extensionalist give for rejecting it? A full defence of the rejection of anti-symmetry is beyond the scope of this paper; however, it may be helpful to note a few relevant points. The first is that Varzi himself appears to think that the rejection of anti-symmetry is a genuine option for mereology:

... concerning the anti-symmetry postulate ... in this case there is a legitimate concern that one of the principles that we are assuming to be constitutive of 'part' is in fact too restrictive. ${ }^{12}$

This is particularly striking given the fact that Varzi states that his argument in defence of extensionality principles does not presuppose anti-symmetry.

${ }^{10}$ Very little is known about non-well-founded mereology, so it may well be that there is a stable position which rejects the inference in step (b). Varzi himself has some sympathy for non-well-founded mereology: see, for example Casati and Varzi, Parts and Places: the Structures of Spatial Representation, p. 36. A non-well-founded relation that allows things to be proper parts of themselves will not, in the end, be definable using ( $\mathrm{PPI}$ ); but there are other options. There may be some independent reason for anti-extensionalists to choose this route, given that nonwell-founded set theories are relatively well studied. See P. Aczel, Non-Well-Founded Sets (Stanford: CSLI Publications, I988); J. Barwise and L. Moss, Vicious Circles: on the Mathematics of Non-Well-Founded Phenomena (Stanford: CSLI Publications, I996).

11 This assumes that our mereology is atomic. Non-atomic mereologies have infinite descending parthood chains, and so are non-well-founded in a different way: see, e.g., A. Tarski, 'Foundations of the Geometry of Solids', in his Logic, Semantics, Metamathematics (Oxford: Clarendon Press, I956), pp. 24-30.

12 Casati and Varzi, Parts and Places: the Structures of Spatial Representation, p. 36. This admission is in part a response to D.H. Sanford, 'The Problem of the Many, Many Composition Questions, and Naïve Mereology', Noûs, 27 (I993), pp. 219-28, who contends that the naïve notion of parthood does not require anti-symmetry. Sanford cites Borges' 'Aleph' as one example. While Varzi concurs with P. van Inwagen, 'Naïve Mereology, Admissible Valuations, and Other Matters', Noûs, 27 (1993), pp. 229-34, that fictional examples do not constitute very weighty evidence, he does think that non-well-founded set theories provide some independent justification for rejecting anti-symmetry. 
The second relevant anti-extensionalist reason for rejecting anti-symmetry comes from considerations about extensionality itself. There is a parallel between (EP) and the anti-symmetry axiom. (EP) says that if $x$ and $y$ are composite objects with the same proper parts, then $x=y$. Anti-symmetry says that if $x$ and $y$ are objects with the same improper parts, then $x=y$. That is, there is an important sense in which the antisymmetry axiom just is an extensionality principle for improper parthood. ${ }^{13}$ Improper parthood, for those against anti-symmetry, is not simply identity. Given that the parthood relation is given as a primitive, one can give only a rough and informal characterization of the notion of improper parthood. But the basic idea is that improper parts are mereologically equivalent: they are indistinguishable using purely mereological predicates, but distinguishable using non-mereological ones. If one assumes that mutual parts are topologically indistinguishable as well, then improper parthood can be thought of as a kind of collocation.

One might think it plausible that although the lump of clay and the statue are not identical, they are completely collocated and 'made up of' exactly the same matter. They might not only be thought of as having all the same proper parts: in addition the anti-extensionalist can plausibly claim that they have all the same parts generally, including improper parts. They are 'mutual parts': the clay is an improper part of the statue and the statue is an improper part of the clay.

The final relevant point is that there are in fact philosophers who defend similar positions. Indeed, this is an important part of Judith Thomson's solution to puzzles of constitution. ${ }^{14}$ Thomson thinks that the statue is constituted by the clay, and that constitution requires mutual parthood: ' $x$ constitutes $y$ at $t$ only if $x$ is part of $y$ at $t$ and $y$ is part of $x$ at $t^{\prime} .{ }^{15}$ Constitution, Thomson thinks, does not imply identity; hence she rejects the anti-symmetry axiom.

\section{OTHER EXTENSIONALITY PRINGIPLES}

Varzi defends in 'The Extensionality of Parthood and Composition' two other extensionality principles: the uniqueness of composition and the extensionality of composition. It will be worth considering whether they too fail when anti-symmetry fails. If they hold in the absence of anti-symmetry, then the anti-extensionalist rejection of antisymmetry will not by itself be a successful response to Varzi's argument. But if they fail where anti-symmetry fails, then it is apparent that Varzi's defence of extensionality principles heavily depends on the acceptance of anti-symmetry.

The next version of extensionality is sometimes called the uniqueness of composition principle:

UC. If $x$ and $y$ are sums of the same things, then $x=y$.

Here by 'sum' I simply mean the mereological relation given by (S) below:

13 Thanks to Achille Varzi for this point.

14 See J.J. Thomson, 'Parthood and Identity Across Time', Journal of Philosophy, 8o (1983), pp. 20I-20, at pp. 208, 219 fn. I2.

15 Thomson, 'The Statue and the Clay', Noûs, 32 (I998), pp. I49-73, at p. I55. 
S. $\quad x$ is a sum of the $z \mathrm{~S} \equiv_{d f}$ the $z \mathrm{~s}$ are all parts of $x$ and every part of $x$ has a part in common with at least one of the $z \mathrm{~s} .{ }^{16}$

As Varzi shows (p. IIo), (UC) implies the extensionality principle (EP). I have already shown that where anti-symmetry fails we can have counter-examples to (EP). But the failure of (EP) implies the failure of (UC) by modus tollens. In any case, the model given in Fig. I is also a counter-example to (UC). $a_{1} \neq a_{2}$. But they are both sums of $b_{1}$ and $b_{2}$. Why? Because both $b_{1}$ and $b_{2}$ are parts of $a_{1}$. The only other parts of $a_{1}$ (namely, $a_{1}$ and $a_{2}$ ) have parts in common with $b_{1}$ and $b_{2}$. The same argument can be run for $a_{2}$.

In addition, classical mereology includes the extensionality of composition principle

EC. If $x$ and $y$ are composed of the same things, then $x=y$.

Here 'composed' is a technical term defined by

C. $\quad x$ is composed of the $z \mathrm{~s} \equiv_{d f} x$ is a sum of the $z \mathrm{~s}$ and the $z$ s are pairwise disjoint.

The difference between composition, given by $(\mathrm{C})$, and sum, given by $(\mathrm{S})$, is that composition requires that the $z \mathrm{~s}$ composing some object must be pairwise disjoint, whereas in a mereological sum of the $z \mathrm{~s}$, the $z \mathrm{~s}$ can overlap. So even though every object composed of the $x$ s is also a sum of the $x$ s, not every sum of the $x \mathrm{~s}$ is composed of those $x \mathrm{~s}$. In Figure 2, $a_{1}$ is a sum of $b_{1}$ and $b_{2}$, but is not composed of $b_{1}$ and $b_{2} ; a_{1}$ is composed of $c_{1}, c_{2}$ and $c_{3}$. As Varzi shows

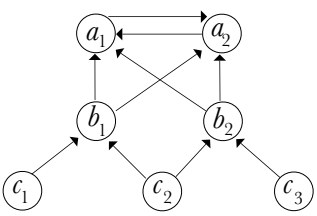

Figure 2 (p. IIo), (EG) does not imply (EP). However, counterexamples to (EC) are available as a result of denying anti-symmetry. One such counter-example is represented in Fig. 2. According to the digraph, $a_{1}$ and $a_{2}$ are mutual parts composed of $c_{1}, c_{2}$ and $c_{3}$. That is, $a_{1}$ and $a_{2}$ are both sums of $c_{1}, c_{2}$ and $c_{3}$ which are themselves pairwise disjoint. But $a_{1} \neq a_{2}$, and so we have a counterexample to (EG).

By denying anti-symmetry - the extensionality principle for improper parts - the anti-extensionalist has counter-examples to all the standard mereological extensionality principles, as desired. This points to a unified response to extensionality principles in mereology.

\section{CLASSICAL MEREOLOGY WITHOUT EXTENSIONALITY}

What kind of mereology would relaxing the anti-symmetry requirement yield? How much of classical mereology must the anti-extensionalist give up as a result of denying anti-symmetry? In this section, I outline a non-anti-symmetric mereology. But importantly, I show how the anti-extensionalist can preserve all of classical mereology without incurring the costs of extensionality principles.

16 This relation can be formulated without plural quantification, but I stick to Varzi's formulation here and below: Varzi, 'The Extensionality of Parthood and Composition', p. Iog. 
The formal picture is grounded on the philosophical intuition that mutual parts are, in some sense, 'mereologically equivalent'. They have all the same (proper and improper) parts, and so their mereological structure is the same. Varzi in 'The Extensionality of Parthood and Composition' calls this 'mereological indiscernibility'. The extensionality principles of mereology turn on the claim that if two objects are mereologically indiscernible, then they are identical. This conditional is precisely what anti-extensionalists reject. I aim to show that by making mereological equivalence explicit, we can recover classical mereological structure.

If the anti-extensionalist opts to deny anti-symmetry, this has a neat formal consequence: being reflexive and transitive, the parthood relation constitutes a preorder $\lesssim$ on the domain of objects $D$. But any preorder can be turned into a partial order. How? We first define a relation $\sim$ :

Sim. $x \sim y=_{d f} x \leqq y$ and $y \leqq x$.

This relation represents our notion of mutual part. ${ }^{17}$ But it is easy to show that $\sim$ is an equivalence relation on $D$. For reflexivity: $x \sim x$ iff $x \leqq x$ and $x \leqq x$. But since $\lesssim$ is reflexive, so is $\sim$. For symmetry: assuming $x \sim y$, we have it that $x \leqq y$ and $y \leqq x$, and so we have it that $y \sim x$. For transitivity: assuming that $x \sim y$ and $y \sim z$, we have it that $x \leqq y, y \leqq x, y \leqq z$, and $z \leqq y$. So by the transitivity of $\lesssim, x \leqq z$ and $z \leqq x$. So $x \sim z$.

So the relation $\sim$ forms equivalence classes out of objects in $D$ that are mutual parts. Informally, treats mutual parts as equivalent or as mereologically indiscernible, while strictly speaking they are not identical. If we take the set $D / \sim$ of all these equivalence classes as a new domain, the parthood relation which was originally a preorder on $D$ will now be a partial order on $D / \sim$. I shall call this resulting partial order ' $\leq$ '.

How this works is shown by the way in which $\sim$ partitions the $D$ into equivalence classes. Where $z$ is some object that has no mutual parts, the equivalence class of $z$, $[z]$, is the set containing $z,\{z\}$. So the equivalence classes only make a formal difference with respect to mutual parts; they only matter where anti-symmetry fails. The right-to-left direction of the definition of $\sim$ above states that if $x$ and $y$ are mutual parts, then $\sim$ treats them as equivalent. So in the new domain, $x$ and $y$ will be in the same equivalence class, even though not identical. As a result, antisymmetry can now be proved to hold:

Anti-symmetry*. If $x \leq y$ and $y \leq x$, then $[x]=[y] .{ }^{18}$

The axiom does not say that objects with the same improper parts are identical. It says only that such objects are in the same equivalence class; they are treated as mereologically equivalent. Between mereologically equivalent objects $x$ and $y$ the

${ }_{17}$ P.M. Simons, in Parts: a Study in Ontology, considers a similar relation as a 'superposition' relation. See p. II for his brief discussion.

${ }^{18}$ Here and below, I simplify the axioms to include equivalence classes only where they are relevant. None of this is particularly significant, since we have it that $x \leqq y$ in $D$ iff $x \leq y$ in $D / \sim$. To show as much, it suffices to show that $\leq$ respects arbitrary witnesses: $a \leq c$ iff $b \leq d$ where $a \sim b$ and $b \sim c$. As can be checked, this falls out of the definition of $\sim$ and the fact that $\lesssim$ is reflexive and transitive on $D$. 
indiscernibility of identicals does not apply simply because $x$ and $y$ are not identical. So the philosophically objectionable result does not follow that everything true of $x$ is true of $y$, nor that everything true of $y$ is true of $x$.

Moreover, again there is a choice in defining proper parthood:

PPI*. $x$ is a proper part of $y \equiv_{d f} x \leq y$ and $[x] \neq[y]$

$\mathrm{PP}_{2} * x$ is a proper part of $y \equiv_{d f} x \leq y$ and $y \nless x$.

But now that I have abstracted away from non-mereological differences, it turns out that $\left(\mathrm{PPI}^{*}\right)$ and $\left(\mathrm{PP}_{2} *\right)$ are equivalent. Why? The second conjunct of $\left(\mathrm{PPI}^{*}\right)$ is $[x] \neq[y]$. We know that $[x] \neq[y]$ if and only if $x+y$. But $x+y$ is by definition equivalent to 'either $x \notin y$ or $y \notin x$ '. This implies that either $x \leq y$ or $y \leq x$ (proved as above). But the first conjunct of (PPI) rules out the case where $x \neq y$. So ( $\left.\mathrm{PPI}^{*}\right)$ is equivalent to ' $x \leq y$ and $y \leq x^{\prime}$. This is the definition of proper parthood given by $\left(\mathrm{PP}_{2}^{*}\right)$.

It is now guaranteed that all of the classical mereological structure is consistent in the new domain. This includes all the standard supplementation axioms, unrestricted fusion, and the like. In fact, if (i) every element in $D$ (except the whole universe) has a complement in $D, 19$ and (ii) every non-empty subset of $D$ has a $\lesssim$-minimal upper bound, ${ }^{20}$ then adding anti-symmetry will be a classical mereo$\operatorname{logy} .{ }^{21}$ But if (i) and (ii) hold in $D$, (i) and (ii) also hold in $D / \sim$; so in that case it is guaranteed that $D / \sim$ is a classical mereology.

One might have noticed that this structure will return all the extensionality principles. But it is crucial to see that these principles are now slightly changed:

EP*. If $x$ and $y$ are composite objects with the same proper parts, then $[x]=[y]$

UC*. If $x$ and $y$ are sums of the same things, then $[x]=[y]$

EC*. If $x$ and $y$ are composed of the same things, then $[x]=[y]$.

As pointed out above, these principles are unobjectionable to the anti-extensionalist. Mereologically equivalent objects can differ along many (non-mereological) properties. In my earlier example, while the lump of clay and the statue are mereologically equivalent, they can still have many different properties without entailing failures of $\left(\mathrm{EP}^{*}\right),\left(\mathrm{UC}^{*}\right)$ or $\left(\mathrm{EC}^{*}\right)$. In an important sense, then, the anti-extensionalist who rejects anti-symmetry is free to endorse a classical mereology, without thinking that 'mereological equivalence' entails identity.

Indeed, the anti-extensionalist can agree that classical mereology is almost entirely correct, with the sole exception that it runs together mereological equivalence and identity. As was shown in §II, denying anti-symmetry is sufficient for losing all extensionality principles. The fact that $D / \sim$ is a classical mereology shows that the only result of denying anti-symmetry is losing extensionality principles.

${ }^{19}$ By complement, I mean a Boolean complement. This can be guaranteed by stipulating that $D$ satisfies the following axiom: if $x$ is not part of $y$ then there is some $z$ that comprises all and only the parts of $y$ that are disjoint from $x$.

${ }^{20} \mathrm{I}$ call $s$ a $\lesssim$-upper bound of $S \subseteq D$ if for every $x \in S, x \lesssim s$. So I say $s$ is a $\lesssim$-minimal upper bound of $S$ if for every $y$ such that $y$ is a $\lesssim$-upper bound of $S, s \leqq y$.

${ }_{21}$ This result can be found in P. Hovda, 'What is Classical Mereology?', Journal of Philosophical Logic (forthcoming), http://dx.doi.org/ıo.I007/siog92-008-9092-4. 
Finally, towards axiomatizing the mereology present in $D$, I note that the resulting partial order $\leq$ on $D / \sim$ is expressible in $D$ via the following definition: $x \leq^{*} y \equiv_{d f} \forall w \forall z(w \sim x \wedge z \sim y \rightarrow w \leqq z)$. One can also define identity in $D / \sim$ thus: $x=* y \equiv_{d f} x \sim y$. One can then translate the classical mereological axioms (including the supplementation axioms and unrestricted fusion) using $\leq^{*}$ and $=*$ to generate an axiomatization for the non-anti-symmetric mereology present in $D .{ }^{22}$

I have argued that Varzi's defence of extensionality principles does depend on the anti-symmetry of the parthood relation. I have shown how two definitions of proper parthood are not equivalent unless anti-symmetry is assumed, and that Varzi's argument crucially depends on the definition which is less plausible by the antiextensionalist's lights. Given the more plausible definition, the argument is not valid unless anti-symmetry is presupposed. I have suggested that there are independent reasons for rejecting anti-symmetry as a response to extensionality principles. Finally, I have given a formal sketch of one way for the anti-extensionalist to recapture full classical mereology without having to accept the extensionality principles or the corresponding 'failures' of the indiscernibility of identicals. As a result, I hope to have given one viable line of response to Varzi's defence of the extensionality of parthood and composition. ${ }^{23}$

\section{University of Connecticut}

22 Thanks to Andrew Bacon for discussion here.

23 Thanks to Andrew Bacon, Don Baxter, JC Beall, Colin Caret, Reed Solomon and Achille Varzi for valuable comments and discussion. 